\title{
MULTIAGENT BASED MULTIPATH ROUTING IN WIRELESS SENSOR NETWORKS
}

\author{
Vidya S. Bennur ${ }^{1}$, Sadashiv S. Shirabur ${ }^{2}$ and Ashok V. Sutagundar ${ }^{3}$ \\ ${ }^{1}$ Department of Electronics and Communication Engineering, East Point College of \\ Engineering for Women, Bengaluru, Karnataka, India - 560049 \\ ${ }^{2}$ Department of Electronics and Communication Engineering, Veerappa Nisty \\ Engineering College, Shorapur, Karnataka, India - $585220 \quad{ }^{3}$ Department \\ of Electronics and Communication Engineering, Basaveshwar Engineering College \\ Bagalkot, Karnataka, India - 587102
}

\begin{abstract}
This paper proposes a Multiagent Based Multipath Routing (MBMR) using a set of static and mobile agents by employing localization technique. The operation of proposed routing technique can be briefly explained as follows. (1) Anchor nodes are deployed evenly over the network environment. (2) Unknown sensor nodes are deployed randomly over network environment and these nodes perform localization. (3) Source node computes the shortest route to destination node through arbitrary midpoint node and intermediate nodes. (4) Source node generates mobile agents for partial route discovery, which traverses to destination node through the midpoint and intermediate nodes by carrying information. (5) Mobile agents update the destination node with carried information. (6) Destination node computes route weight factor for all the routes discovered by mobile agents. (7) Destination node computes the node disjoint routes and it selects routes depending on the criticalness of event for communication. The performance of the proposed scheme is evaluated in terms of performance parameters such as localization error, network lifetime, energy consumption, cost factor, packet delivery ratio, and latency.
\end{abstract}

\section{KEYWORDS}

Wireless Sensor Networks, Localization, Agents, Multipath Routing

\section{INTRODUCTION}

In the recent years, the advances in VLSI technology, Micro-Electro-Mechanical (MEMS) system and low power radio technologies have created low power, low cost and multifunctional wireless sensor devices, which can observe and react to changes in physical phenomenon of their environment. WSNs consist of a very large number of small, inexpensive, disposable, robust and low power sensor nodes working co-operatively. The sensor node consist of sensing unit to sense the environment, processing unit to process the sensed raw data, memory, battery and transceiver for communication [1-4]. These sensor nodes are scattered across the geographical area with larger number nodes to sense over an area so that they can provide greater accuracy. Typically the sensor nodes coordinate themselves to perform a common task, and are capable of collaborating with one on another and measuring the condition of their surrounding environment. The sensed measurements (data) are then transformed in to digital signal and processes to reveal some properties of the phenomenon around the sensors. Due to the fact that sensor nodes in WSNs have short radio transmission range, the intermediate nodes act as relay nodes to transmit data towards the destination node (sink node). The sensors can be used to monitor wide variety of ambient conditions (parameters) such as temperature, humidity, pressure, DOI : 10.5121/ijwmn.2014.6207 
salinity and mobility. This monitoring capacity of sensors can be effectively used in applications like military (battlefield surveillance) application, environment (fire detection, earthquake monitoring) application, agriculture irrigation monitoring, and health applications [5].

We briefly describe the different types of wireless networks such as cellular and ad-hoc networks in order to differentiate the WSNs from them. The cellular network is one which consists of cellular phones those are mobile devices and roaming over an area, the area is divided into smaller regions called as cell and each cell is equipped with a Base Station (BS). The BS of all the cells is connected to a backbone network. The mobile devices communicate each other by establishing a connection through the BS. The cellular network uses packet switching mode operation in recent days, but traditionally it uses circuit switching. The ad-hoc network is an infrastructureless network because the sensor devices are deployed over an area without the infrastructure. All the devices are mobile and communication is relayed on many mobile devices so each device can act as user and router for data transmission. These networks are established in an emergent situation over a small geographical area. However the mobile devices are rechargeable. Sensor networks consists of large number of nodes in the network, deployed densely which can be several orders of magnitude higher than the nodes in an ad-hoc network. Sensor nodes are more prone to failures. Topology of sensors network changes very frequently compared to ad-hoc network. Sensor nodes mainly use broadcast communication paradigm than point to point communication. Sensor nodes are limited in power, computation capabilities, memory and most of the time batteries of the nodes are not rechargeable [6].

In traditional WSNs, the sensors nodes continuously at a periodic time gather the data and transmit it to the destination node by multi-hop communication. The conventional method of data gathering and processing in WSNs may lead to some of the limitations like increased latency, excess energy consumption, bandwidth overhead and redundant data transmission etc. The important issue in WSNs is the network lifetime which is mainly dependent on the energy of sensor nodes.

Recently, the multipath routing is widely used by many researchers to develop the best networking protocols to improve the network performance by utilizing the network resources effectively. As the radio transmission range of sensor node is short, the communication between the source and destination is accomplished by multi-hop communication. But the communication cost is usually several orders of magnitude higher than the computation cost. During routing phase, the sensed data, query and control messages travel from a node to another node to reach the destination node. This transfer consumes a considerable amount of sensor energy. Therefore many routing protocols are proposed for conserving the sensor energy and to prolong the lifetime of network. But many of these protocols use conventional method for the route discovery which also consumes a considerable amount of sensor energy.

Agents have been used in many applications to reduce the number of message that need to be send from one sensor to another. This paper proposes the routing protocol in WSNs using agents to reduce the number of data and control messages and prolong the sensors lifetime. Agents are also great tools in case of communication with low bandwidth which is one of the main features of sensor networks.

The WSNs are large scale networks with hundreds and even thousands of highly resource constrained sensors nodes put together in network. For example in a network, sensors are densely distributed across a large building or over an area such as forest, the typical tasks for such a network are, to send a data to a sensor which may be a destination node, but this task requires the knowledge of position of the sensor nodes or at least relative positions of sensor nodes. To know the position of each sensor node it is possible to equip the each sensor node with a Global Position System (GPS) which provides them with absolute position, but this is currently a costly solution. Because in WSNs the energy conservation has been considered as a core issue and cost, as well as size of the sensor node should be as small as possible. So these constrains of WSNs is not overcome just by equipping GPS to each sensor node. 
International Journal of Wireless \& Mobile Networks (IJWMN) Vol. 6, No. 2, April 2014

Unfortunately, for a large scale network with hundreds or thousands of sensor nodes the solution is not adding GPS to all sensor nodes in the network and it's not feasible because [7]. 1) GPS consumes more energy for its operation which inturn reduce the life time of WSN. (2) In large scale WSN if each sensor node is equipped with the GPS, then the production cost of the network will be very high. (3) Size of sensor nodes are required to be small but the size of the GPS and its antenna increases the sensor node size. (4) In the presence of dense forests, mountains or other obstacles that block the lineof-sight from GPS satellites, GPS cannot be implemented.

For these reasons an alternate solution of GPS is required which is cost effective, energy conservable, effective in sensor size and can operate in diverse environments. The multipath routing algorithm proposed in this paper requires sensor nodes to be able to locate themselves in co-ordinate system in various environments. This problem, to which we refer as localization, is a challenging issue in WSNs to improve the quality of network in terms of energy consumption, size and cost [8][9][10]. The localization opens up new ways of reducing power consumption in multi-hop WSNs. To perform the localization, the proposed routing protocol uses the power of the received beacon signal of the three anchor nodes and with the aid of trilateration method the position of node is determined.

Many of the subsisting research presented the routing protocol for WSNs which are dealing with: energy awareness to maximize the lifetime of WSNs, fault tolerance in case of nonworking sensor nodes and scalability for large number of sensor nodes. Some of the critical application such as fire detection, disaster management and military application etc need energy constraint, delay constraint routing, size of the sensor nodes as small as possible and network production cost should be as small as possible.

\subsection{Related Work}

Some of the related works of localization technique are as follows: Estimating the geographic position of sensor node is a crucial issue in WSNs to reduce the power consumption, size and cost of wireless device without equipping more of sensors with GPS. The localization from mere connectivity presents a method to determine the location of nodes in the network by using the connectivity information [11]. It uses multidimensional scaling to determine the location of nodes.

The work given in [12] describes a localization algorithm based on the angle of arrival of beacons from three or more fixed beacon nodes. In this technique to reduce the hardware complexity it uses direction estimation of received beacons. The beacon node location and these angular directions are used by standard triangulation technique to determine location of sensor node. Ad-hoc positioning system uses angle of arrival capability of nodes with triangulation technique to determine the orientation and position of nodes in ad-hoc network [13].

A multi-hop localization technique for WSNs using acquired Received Signal Strength Indication (RSSI) is presented in [14]. In this technique the RSSI packet is exchanged between the nodes and based on this a ranging model is constructed then it is used by minimum least square algorithm to find location of node. The work given in [15] describes a GPS less localization scheme for outdoor environment based on connectivity metric. The connectivity metric uses the Radio Frequency (RF) communication capabilities of wireless devices. A fixed number of reference points are used for localization with overlapping coverage regions and transmit periodic beacon signals. Nodes use a simple connectivity metric to localize themselves to nearest reference points.

The fully decentralized Anchor Free Localization (AFL) algorithm is presented in [16]. The nodes in AFL start from a random initial co-ordinate assignment and converge to a consistent solution using only local node interaction. The robust positioning algorithm for distributed ad-hoc WSNs is depicted in [17]. The localization algorithm works in two phases, in first phase it uses hop-terrain algorithm with triangulation to find the node position. In second, refinement phase the more accurate position of node is computed by least square triangulation technique which is an iterative algorithm. A practical localization algorithm for WSNs is presented in [18]. It works in two phases, first, it partition the 
target region by dividing into small grids. Second, the refinement phase in which more accurate location of node is determined by applying trick algorithm.

The work given in [19] describes an improvement to Distance vector (DV)-hop algorithm. It presents a new DV-hop algorithm based on optimal nodes. A RSSI based DV-hop algorithm for WSNs is depicted in [20]. For localization each node uses three or more beacon signals from anchor nodes. But in this algorithm each sensor node uses hop count from two anchor nodes and RSSI of another anchor node for location identification. The work in [21] describes the location tracking in indoor environment using the RSSI of beacon nodes with trilateration estimation technique.

Some of the related works of multipath routing are as follows: Energy based multipath routing protocol for WSNs describes how the network life time can be maximized through balancing energy consumption across multiple sensor nodes [22]. It uses service differentiation concept to provide the accepted delay for delay sensitive application. This protocol employs a queuing model to differentiate between real and non-real time traffic. It reduces the end-to -end delay by distributing traffic across multiple paths and increases the throughput rate to shortest path.

The work given in [23] presents the awareness of Quality of Service (QOS) and energy efficiency by multipath routing which increases the network lifetime in WSNs. It establishes a tradeoff between energy and QOS to improve the network performance. Relax multipath routing protocol for WSNs is presented in [24]. This protocol uses battery relaxation period to improve the network lifetime and reliability. The relaxation period is used to recover the battery from its lost capacity to increase the battery life, which increase the lifetime of entire network. To provide reliable service, the source node periodically floods the keep alive message over multiple paths to keep them alive.

The [25] describes the energy efficient and QOS based routing protocol for WSNs. It achieves load balancing through splitting up the traffic across a set of available node disjoint paths. This protocol increases the reliability of data delivery through utilization a light wait XOR based foreword error correction technique to provide data redundancy. It uses the residual energy; buffer size and signal to noise ratio to predict the next hop through the path construction phase.

The congestion control in WSNs based multipath routing in priority rate adjustment technique protocol is presented in [26]. The congestion in WSNs needs to be controlled to prolong the lifetime of network and to avoid packet loss. It is required to improve the fairness and provide better QOS. The efficient multipath protocol for WSNs describes a multipath routing algorithm based on direct diffusion that reinforces multiple paths with link quality and low latency [27]. It improves the energy efficiency and scalability.

When certain nodes and links become over utilized causes congestion, the traffic can be spread over the alternative paths to balance the load over those paths and increases the degree of fault tolerance. The work given in [28] describes design of an alternate multipath routing protocol for WSNs when the nodes on primary path fail. The work given in [29] depicts a design of a multipath energy efficient congestion control scheme to reduce the packet loss due to congestion. It presents accurate detection of congestion and its control. For congestion estimation it uses combination three parameters queue size, contention and traffic rate. To control the congestion it uses two techniques depending on degree of congestion defined by three parameters, which are rate control technique and multiple energy efficient paths.

To enable reliable data delivery a multipath routing algorithm is presented in [30]. The packet loss and congestion in the network can be reduced by controlling the scheduling rate of packets depending on priority of data. The fault diagnosis is a important issue in WSNs to reduce energy consumption by faulty nodes and to ensure the gathering information accuracy. An energy efficient data collection protocol which consists of clustering and multipath routing is described in [31]. The clustering based on fault diagnosis avoids the faulty nodes becoming a cluster heads so that it reduce the energy consumption and fault information transmission. 
International Journal of Wireless \& Mobile Networks (IJWMN) Vol. 6, No. 2, April 2014

The work given in [32] describes that continuously using the minimum energy path deprives the nodes energy and time taken to determine an alternate path increases. The energy efficient adaptive multipath routing technique is proposed, the adaptive is because they have low routing overhead. This protocol provides reliability and low energy consumption. Agent based multipath routing presented in [33] which describe the use of agents to reduce the complexity in routing technique. The agents also reduce the communication cost.

The work given in [34] describes the data gathering and aggregation to avoid the redundant data transmission and to reduce the bandwidth required for data transmission. To perform aggregation of data it constructs a wheel with spokes around the event triggered node. The energy consumption reduced by using the agents for path discovery. It provides reliability for data transmission by multiple paths.

Some of the drawbacks of existing localization technique are as follows: lack of mechanisms for anchor node deployment, time of arrival based localization technique needs extra hardware otherwise a small error in time causes larger error in distance estimation and angle of arrival based localization technique needs extra directional antenna and it is not advisable for large WSNs. And drawbacks of existing routing protocols are: use of GPS to all nodes increases the size of wireless device, cost and energy consumption which leads to reduced network lifetime, conventional method of route discovery and route maintenance consumes more energy, lack of robust mechanism for critical information transmission, lack of mechanism for selecting good relay nodes for route construction.

We have proposed a MBMR in WSNs by employing localization. The localization technique works based on the received power of beacon packet from anchor nodes. These beacons are prioritized based on their power level and three highest power beacons are used to distance estimation with appropriate propagation model. Due to use of localization scheme the size of the some nodes is reduced and cost of network deployment is also reduced. As the GPS is used only by anchor nodes the energy consumption by GPS in unknown nodes is reduced.

\subsection{Our Contribution}

This paper proposes MBMR scheme in WSNs. The proposed scheme uses two types of software agencies which are sensor and destination node agencies and those are static and mobile.

The operation of the proposed routing scheme can be explained as follows: (1) Anchor nodes are deployed evenly over the entire network. (2) Unknown sensor nodes are randomly deployed over the entire network environment. (3) Now each anchor node broadcasts a beacon signal by flooding (up to 2 hops). (4) Unknown sensor nodes perform localization to know its location co-ordinates. (5) Whenever a sensor node detects an event, than it announces that, it is the source (event) node to its neighbors and it computes the shortest distance to destination node using GPS location information and its location co-ordinates. (6) Source node computes the arbitrary midpoint node between source node and destination node using Euclidean distance. It also determines the intermediate nodes above and below the midpoint node and also calculates the ascending and descending angles. (7) Source node generates mobile agent and its child; each one will move on midpoint node and intermediate node above and below the midpoint node by carrying the information like node id, residual energy, hop count and hop distance, event information etc. (8) The mobile agent and its child which are traversed through the network by carrying the route and network information (node id, residual energy, hop count, available bandwidth, hop distance and event information) will updates the destination node with this information. (9) Destination node computes the path weight factor based on hop distance factor, link efficiency and energy efficiency for all the routes discovered by the mobile agents and assign to the respective routes. These routes are prioritized based on their route weight factor and it identifies the node disjoint routes. (10) Destination node evaluates the criticalness of event based on the information carried by the mobile agents. Depending on the criticalness of event 
the destination node selects a single route for in time data delivery or multiple routes for robust and reliable communication. It also triggers mobile agent and its clones to establish the required routes.

Our contributions in the proposed routing technique are as follows: (1) Anchor node deployment evenly over the network environment. (2) Computation of received power from anchor nodes at each unknown sensor nodes. (3) Trilateration technique is applied based on received power at unknown sensor nodes to know their position. (4) Method to select midpoint node and intermediate nodes. (5) Usage of static and mobile agents for route discovery. (6) Route weight factor calculation based on hop distance factor, link efficiency and energy efficiency. (7) Identification of node disjoint route. (8) Best route/s selection based on event criticalness.

\section{MULTIAGENT BASED MULTIPATH ROUTING}

In this section we describe the preliminaries, network environment, models and agencies used for proposed MBMR in WSNs.

\subsection{Preliminaries}

Some of the basic key terms used in proposed scheme are as follows.

a. Anchor node: It is a sensor node which has prior knowledge of its location co-ordinates when it is deployed in the network environment since it is equipped with GPS.

b. Lateration: It is a technique, where the unknown sensor node location is determined through distance measurement from unknown sensor node to anchor nodes.

c. Event: It is an action or occurrence detected by interaction of sensing unit with the environment by sensing or controlling physical parameters and it is worth report to destination (sink) node.

d. Event affected area: It is the area in which sensor node detects an event.

e. Source node: It is the sensor node in the event affected area which detects event first compared to other nodes in that area. It announces itself as a source node to other nodes to avoid the assuming the role of source node by others.

f. Mid point: It is the arbitrary middle point on the straight line joining (reference axis) the source node and destination node.

g. Intermediate nodes: These are sensor nodes located above and below the (reference axis) midpoint node between source and destination nodes and these intermediate nodes are all on the same line with midpoint node.

h. Ascending angle: It is the angle formed between the midpoint node and intermediate node above the reference axis at source node. The agents and messages moves with the ascending angle from source node to intermediate node and with descending angle from intermediate node to reach the destination node.

i. Descending angle: It is the angle formed between the midpoint node and intermediate node below the reference axis. The agents and messages moves with the descending angle from source node to intermediate node and with ascending angle from intermediate node to reach the destination node.

j. Hop count: It is the total number of relaying nodes between the source node and destination node.

\subsection{Network Environment}

The network environment is shown in figure (1), which consists of tiny sensor nodes with heterogeneous sensing capability and destination node. In this network environment the sensor nodes are classified in two types which are unknown sensor node and anchor node. The unknown sensor nodes comprises of processor for preprocessing the sensed data, transreceiver for transmission and reception of data and memory. These nodes are deployed randomly over the entire network environment. The anchor nodes comprises of all the components of the unknown sensor node, in 
addition to that it is having a GPS for location awareness. These nodes are deployed evenly over the entire network environment. Immediately after the sensor nodes deployment the localization is performed over the entire network to know the location of unknown sensor nodes by itself. After localization, whenever a sensor node detects an event than it sends data to the destination node by multi-hop communication. We assume that all sensor nodes are stationery for their lifetime and they have same energy during the deployment phase. All the sensor nodes have same transmission range. It is assumed that sensor nodes have capability to reconfigure the transmission power. Each sensor node consists of an agent platform with security feature to coordinate the inter-agent communication.

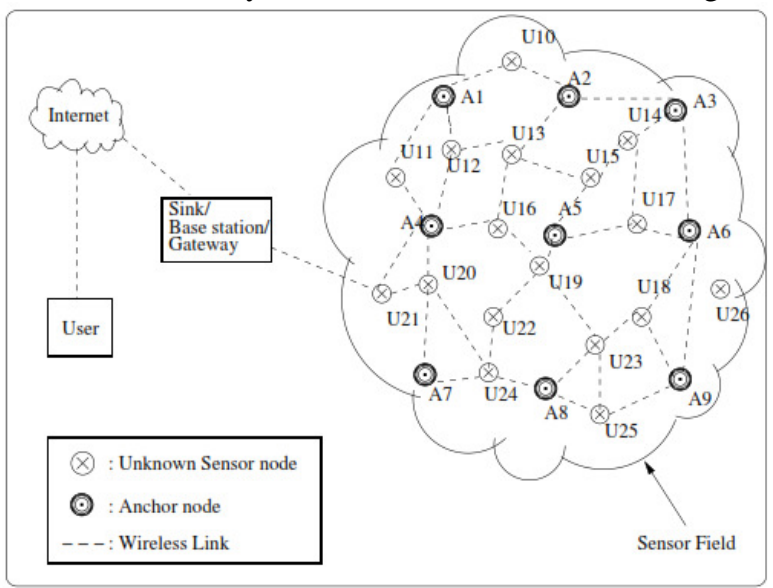

Figure 1: Network Architecture

\subsection{Models}

This section presents the models used for proposed scheme such as anchor node deployment, localization scenario, event detection, path parameters, and partial route discovery and route establishment.

\subsubsection{Anchor Node Deployment}

We consider a $l \times b$ squared area as a network environment for wireless sensor network where $(l=b)$. In this squared area $N_{u}$ identical unknown sensor nodes are deployed randomly, but the $N_{a}$ anchor nodes are deployed evenly over the entire network environment. For performing the localization each unknown sensor node should receive beacon signals from minimum three anchor nodes. Due to this reason the anchor nodes should be deployed at particular locations over the network environment. The anchor nodes can be deployed based on the equation (1) and(2).

$$
\begin{array}{ll}
l_{x}=\frac{l}{z} \times x & \text { Where } x=0,1,2,3, \ldots \ldots \ldots n \\
b_{y}=\frac{b}{z} \times y & \text { Where } y=0,1,2,3, \ldots \ldots \ldots m
\end{array}
$$

Where $l$ is the length and $b$ is breadth of network area, where $(l-b)$ and $\left(h_{x^{\prime}} h_{y}\right)$ gives the deployment location co-ordinates of each anchor node by varying the value of $x$ and $y$ in above equation. The anchor nodes can be deployed up till $\left(l_{x}, b y\right) \leq(l, b)$. The $z$ is a numerical value which should be chosen such that it divides the network area uniformly depending on the number of anchor nodes so that anchor nodes can be deployed evenly over the network. The network environment after the deployment of anchor nodes is shown in figure (2). 


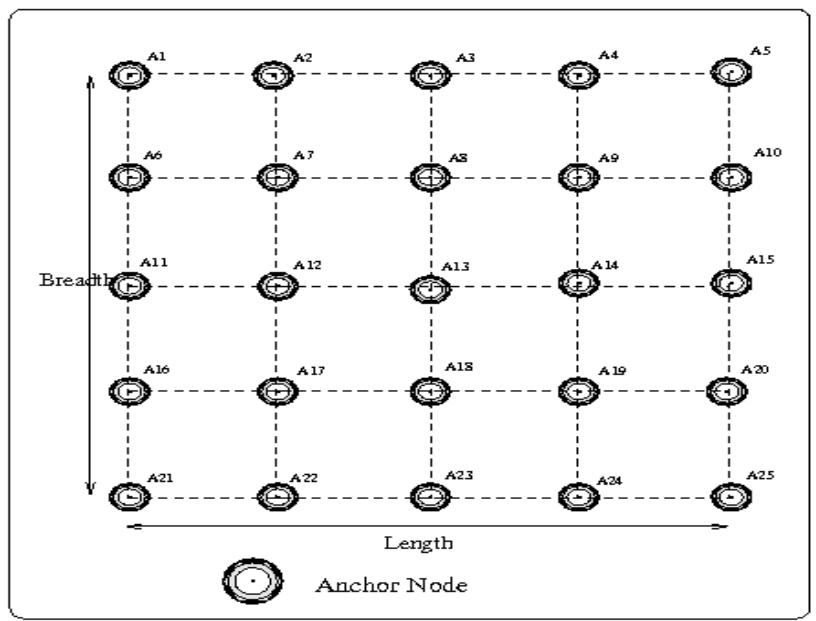

Figure 2: Anchor Node Deployment

\subsubsection{Localization Scenario}

To perform the multipath routing by proposed scheme in WSNs each sensor node in the network should know their location co-ordinates, so to determine the location co-ordinates of unknown nodes the localization is performed, which is as follows. After the deployment of all sensor nodes, each anchor node broadcasts a beacon signal by flooding (up to two hops). The unknown sensor nodes receive the beacon signals from different anchors. We assume that each unknown sensor node receives more than three beacon signal from different anchors. Now each unknown sensor node computes the power received by the reception of every beacon signal using the equations (3) and (4).

$$
\begin{aligned}
& P_{r}=\frac{V_{\mathrm{ms}}^{2}}{R_{a}} \\
& V_{m s}=\frac{V_{p}}{\sqrt{2}}
\end{aligned}
$$

Where $P_{r}$ is the total received power of beacon, $V_{r m s}$ is the rms voltage of the received beacon signal, $V_{p}$ is the peak voltage (maximum of absolute value of signal) of the received beacon signal and $R_{a}$ is receiving antenna resistance. The beacon signals are prioritized based on their received power.

Now the three beacons which are received with maximum power are used to find the distances to their respective anchors from the unknown sensor node. According to empirical propagation measurements in a radio channel shows that the average received signal strength at any point decays as a power law of the distance of separation between a transmitter and receiver. So the distance between the transmitter and receiver can be calculated by using the equation given by ( 5 ).

$$
P_{r}(d)=P_{r}\left(d_{0}\right)-10 n \log \left(\frac{d}{d_{0}}\right)
$$

Where $P_{r}\left(d_{0}\right)$ is power received at distance $d_{0}, d_{0}$ is close in reference distance from the transmitter, $n$ is path loss exponent.

We assume that the close in reference point $d_{0}=1 \mathrm{~m}$ and $P_{r}\left(d_{0}\right)$ can be generally determined by using free space propagation model given by FRIIS transmission formula given by equation $(6)$.

$$
P_{r}=\frac{P_{t} \times G_{t} \times G_{r}}{\left(\frac{4 \pi d}{\lambda}\right)^{2} \times L}
$$

Where $P_{t}$ is transmitted power, $G_{t}$ is transmitter antenna gain, $G_{r}$ is receiving antenna gain, $L$ is system loss factor not related to propagation of data $(\mathbf{L} \geq \mathbb{1})$ ), assume $\mathbf{L}=\mathbb{1}$ (indicates no loss in system hardware), $\lambda$ is wavelength in meters $(c / f)$. 
After estimating the distance to three anchor nodes the trilateration estimation is applied to find the location of unknown sensor nodes. The localization scenario is shown in figure (3) for one unknown sensor node (U8) and same will be performed by each unknown sensor node.

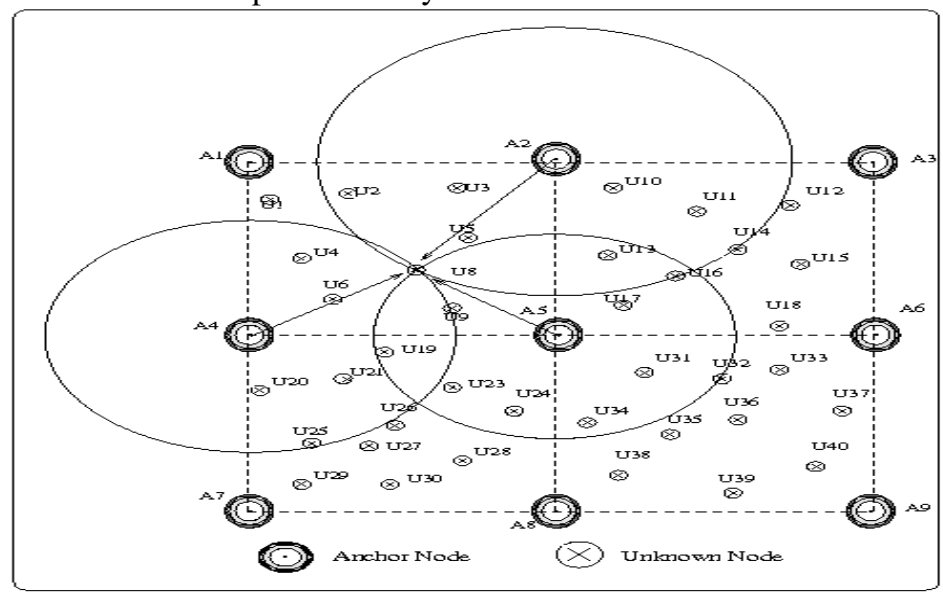

Figure 3: Localization Scenario

\section{Localization Algorithm}

Nomenclature: $\mathrm{P}_{\mathrm{r}}$ : Total power of a received beacon, $\mathrm{N}$ : Number of beacons received by the unknown node, temp : Temporary variable, $\mathrm{i}$ and $\mathrm{j}:$ Index values.

\section{1: Begin}

2: Anchor node broadcast beacon signal;

3: Unknown node receives beacon signals;

4: for $\mathrm{i}=0$ to $\mathrm{N}$ do

5: $\quad$ Calculate $\mathrm{P}_{\mathrm{r}}$;

6: $\quad \mathrm{P}_{\mathrm{r}}[\mathrm{i}]=\mathrm{P}_{\mathrm{r}}$;

7: end for;

8: for $\mathrm{i}=0$ to $\mathrm{N}$ do

9: $\quad$ for $\mathrm{j}=0$ to $\mathrm{N}$ do

10: $\quad$ if $\left(\mathrm{P}_{\mathrm{r}}[\mathrm{i}]<\mathrm{P}_{\mathrm{r}}[\mathrm{j}]\right)$ then

11: temp $=\mathrm{P}_{\mathrm{r}}[\mathrm{i}]$;

12: $\quad \mathrm{P}_{\mathrm{r}}[\mathrm{i}]=\mathrm{P}_{\mathrm{r}}[\mathrm{j}]$;

13: $\quad \mathrm{P}_{\mathrm{r}}[\mathrm{j}]=$ temp;

14: $\quad$ else

15: $\quad$ go to step 9;

16: $\quad$ end if;

17: end for;

18: end for;

19: Use trilateration algorithm;

20: end;

\subsubsection{Trilateration}

The lateration is a most common method for deriving the position co-ordinates of unknown nodes, where the trilateration uses the distance measurements to minimum three anchor nodes. This estimation considers these distances between the reference location (anchor) and unknown location as the radii of circles with centers at every respective reference location (anchor location). Thus the unknown location is the intersection of the three sphere surface as shown in figure (4).

As shown in figure (4) the unknown node $U_{i}\left(x_{i}, y_{i}\right)$ is located using the co-ordinate of anchor nodes $A_{1}\left(x_{1}, y_{1}\right), A_{2}\left(x_{2}, y_{2}\right), A_{3}\left(x_{3}, y_{3}\right)$ and distances between these nodes and 
unknown sensor node $d_{1}, d_{2}, d_{3}$. By using the above information we can derive the following system of equations.

$$
\begin{aligned}
& \left(x_{i}-x_{1}\right)^{2}+\left(y_{i}-y_{1}\right)^{2}=d_{i}^{2} \\
& \left(x_{i}-x_{2}\right)^{2}+\left(y_{i}-y_{2}\right)^{2}=d_{2}^{2} \\
& \left(x_{i}-x_{3}\right)^{2}+\left(y_{i}-y_{3}\right)^{2}=d_{3}^{2}
\end{aligned}
$$

In practice, usually above set of equations has no solution as they are nonlinear. The above set of equations can be converted to linear form of equation by subtracting last equation (9) from first two equations (7) and (8).

$$
\begin{aligned}
& \left(x_{i}-x_{1}\right)^{2}+\left(y_{i}-y_{1}\right)^{2}-\left(x_{i}-x_{3}\right)^{2}+\left(y_{i}-y_{3}\right)^{2}=d_{1}^{2}-d_{3}^{2} \\
& \left(x_{i}-x_{2}\right)^{2}+\left(y_{i}-y_{2}\right)^{2}-\left(x_{i}-x_{3}\right)^{2}+\left(y_{i}-y_{3}\right)^{2}=d_{1}^{2}-d_{3}^{2}
\end{aligned}
$$

Re-ordering the terms in equation (10) and (11) gives a proper system of linear equation as below.

$$
\begin{aligned}
& 2 x_{i}\left(x_{3}-x_{1}\right)+2 y_{i}\left(y_{3}-y_{1}\right)=x_{1}^{2}-x_{3}^{2}+y_{1}^{2}-y_{3}^{2}+d_{3}^{2}-d_{1}^{2} \\
& 2 x_{i}\left(x_{3}-x_{2}\right)+2 y_{i}\left(y_{3}-y_{2}\right)=x_{2}^{2}-x_{3}^{2}+y_{2}^{2}-y_{3}^{2}+d_{3}^{2}-d_{2}^{2}
\end{aligned}
$$

Now the above set of equations (12) and (13) can be represent in the linear matrix form as

$$
A X=B
$$

Where

And

$$
\begin{aligned}
A & =\left[\begin{array}{ll}
2\left(x_{3}-x_{1}\right) & 2\left(y_{3}-y_{1}\right) \\
2\left(x_{3}-x_{2}\right) & 2\left(y_{3}-y_{2}\right)
\end{array}\right] \\
B & =\left[\begin{array}{l}
x_{1}^{2}-x_{3}^{2}+y_{1}^{2}-y_{3}^{2}+d_{3}^{2}-d_{1}^{2} \\
x_{2}^{2}-x_{3}^{2}+y_{2}^{2}-y_{3}^{2}+d_{3}^{2}-d_{2}^{2}
\end{array}\right]
\end{aligned}
$$

$$
X=\left[\begin{array}{l}
x_{i} \\
y_{i}
\end{array}\right]
$$

According to equation(14), the co-ordinate $\left(x_{i}, y_{i}\right)$ of unknown sensor node can be obtained by using estimation method of standard minimum mean square estimation, which is given by equation(18).

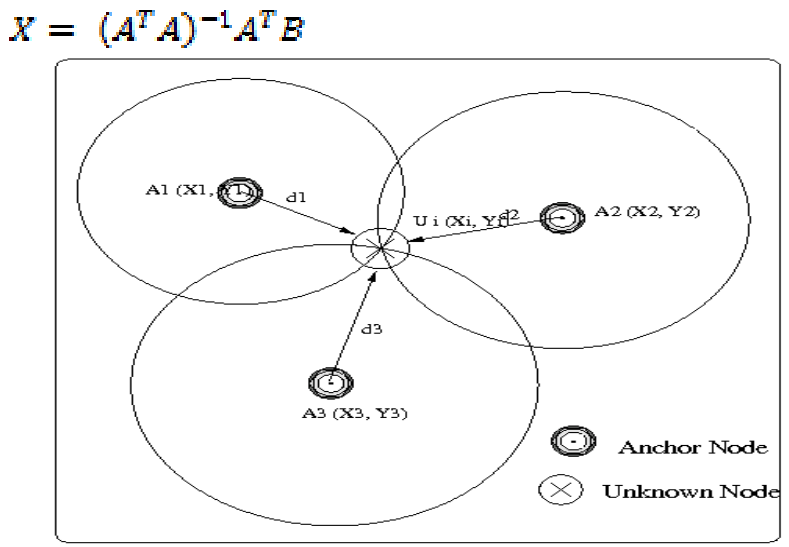

Figure 4: Trilateration

\section{Trilateration Algorithm}

Nomenclature: $\mathrm{N}_{\mathrm{u}}$ : Number of unknown nodes, $\left(\mathrm{x}_{\mathrm{i}}, \mathrm{y}_{\mathrm{i}}\right)$ : Co-ordinates of unknown nodes, $\left(\mathrm{x}_{\mathrm{j}}, \mathrm{y}_{\mathrm{j}}\right)$ : Co-ordinates of anchor nodes, $\mathrm{d}_{\mathrm{j}}$ : Distance between unknown node and respective anchor node, $\mathrm{X}$ : Matrix of co-ordinates of unknown node, $i$ and $j$ : Index values.

1: Begin

2: for $\mathrm{i}=0$ to $\mathrm{N}_{\mathrm{u}}$ do

3: $\mathrm{j}=0$;

4: $\quad$ Calculate $\mathrm{d}_{\mathrm{j}}$;

5: $\quad\left(x_{i}-x_{j}\right)^{2}+\left(y_{i}-y_{j}\right)^{2}=d_{j}^{2} ;$ 


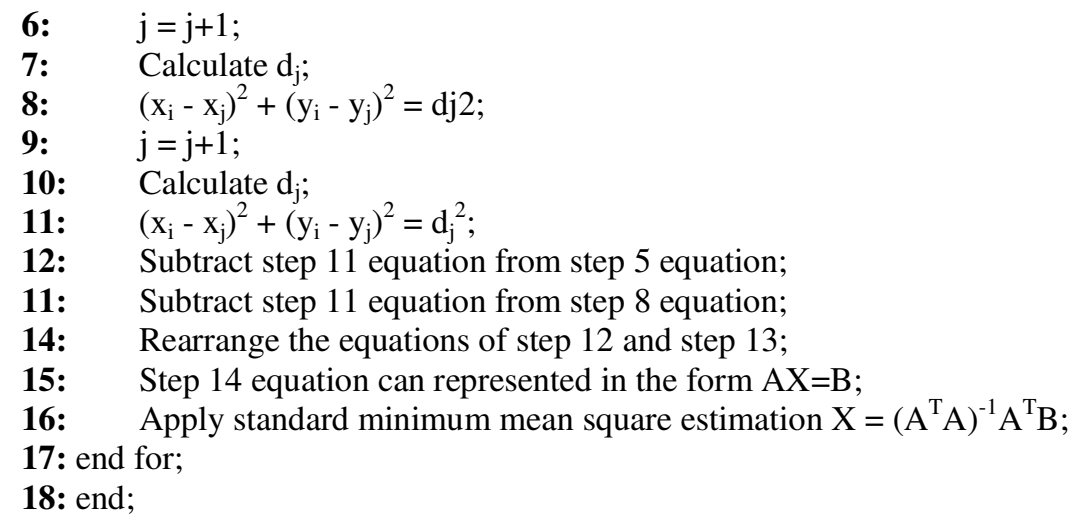

\subsubsection{Event Detection}

The logical relationship between the various parameters sensed by sensor node decides the occurrence of an event. The event detection is performed as follows. Let $g_{1}^{n}, g_{2}^{n}, g_{3}^{n}, \ldots \ldots \ldots, g_{i}^{n}$, be the $n^{\text {th }}$ event with associated sensed parameter values and $g_{n t h 1}^{n}, g_{n t h 2}^{n}, g_{n t h 3 ; \ldots \ldots \ldots}^{n} \ldots g_{n t h i}^{n}$ be the sensor parameter threshold values for event $n$, where $n=1,2,3, \ldots \ldots \ldots, z_{1}$ and $i=1,2,3, \ldots \ldots \ldots j$. The sensed parameter values for the event are either set to 1 or 0 based on event threshold as given by equation $(19) \mathrm{v}_{i=1}^{j}[34]$.

$$
g_{i}^{n}= \begin{cases}1 & \text { if }\left(g_{i}^{n}>g_{n t h i}^{n}\right) \\ 0 & \text { Otherwise }\end{cases}
$$

An occurrence of an event is given by equation $(20)$.

$$
E_{\text {det }}^{n}= \begin{cases}\text { Detected } & \text { if } \\ \text { Notdetected } & \prod_{i=1}^{j} \mathrm{~g}_{i}^{\mathrm{n}}=1 \\ \text { Otherwise }\end{cases}
$$

\section{ROUTE PARAMETERS}

This section depicts the computation of route parameters such as link and route efficiency, energy ratio and hop distance factor used in the proposed scheme.

Link efficiency can be computed as follows. Let $K$ be the discrete time discrete valued channel capacity, $B W$ be the bit rate of the channel, $E_{i}$ be the total energy consumed for transmission of a bit in link $i$ and $S N R$ be the signal to noise ratio [35]. Capacity of channel $i$ is given by the equation(21).

$$
K_{i}=\nabla W \log _{2}(1+s N R)
$$

Let $E_{t}$ be the energy consumed for the transmission of a bit per distance and $E_{i}$ can be computed by using the following equation(22) [35].

$$
E_{i}=E_{t} \times d
$$

Link efficiency $L K$ can be determined by using the equation (23) [36].

$$
L K_{\text {eff }}=\frac{K_{i}}{E_{i}}
$$

Route efficiency can be determined by using the equation (24) by considering $n$ links on the route [37].

$$
R_{e f f}=\operatorname{Min}\left\{L K_{\text {eff } 1}, L K_{\text {eff } 2^{y}} \ldots \ldots \ldots \ldots \ldots K_{\text {ef } j n}\right\}
$$

Energy ratio in each of the route from source node to destination node is computed using the equation (25) and (26) [37].

$$
\begin{aligned}
& R E_{\max }=\operatorname{Max}\left\{R E_{1} R E_{2}, R E_{3, \ldots \ldots \ldots \ldots} \ldots \ldots E_{n}\right\} \\
& R E_{\min }=\operatorname{Mn}\left\{R E_{1}, R E_{2}, R E_{3}, \ldots \ldots \ldots E_{n}\right\}
\end{aligned}
$$


Where $R E_{1}$ to $R E_{n}$ be the residual energy of the $n$ intermediate nodes of a route. The minimum $R E_{\text {min }}$ and maximum $R E_{\text {max }}$ are the residual energy for $n$ nodes in the route computed using above equations $(25),(26)$. Now find route energy efficiency by using the equation $(27)$.

$$
E_{\text {eff }}=\frac{R E_{\text {min }}}{R E_{\text {max }}}
$$

The hop distance factor $\left(H_{d f}\right)$ of a route is determined by using the equation(28).

$$
H_{d f}=\frac{R_{a}}{R_{h o}}
$$

Where $R_{d}$ is the Euclidean distance between source and destination node which is given by the equation (29) and $\boldsymbol{R}_{k c}$ is the total number of hops in route from source node to destination node.

$$
R_{d}=\sqrt{\left(x_{d}-x_{s}\right)^{2}+\left(y_{d}-y_{s}\right)^{2}}
$$

\subsection{Partial Route Discovery}

Partial route discovery is performed by mobile agent (RDA) between the source node and destination node $U_{40}(\mathrm{~S})$ and $D$ respectively. The source node determines the arbitrary midpoint node $U_{2 g}\left(x_{\text {intmm }}, v_{\text {intrm }}\right)$ between source node and destination node with the aid of its locations $\left(x_{g}, y_{s}\right)$ and $\left(x_{d}, y_{d}\right)$ respectively on the reference axis by using the equation (30) and (31) and node $U_{24}$ also lies on the reference axis as shown in figure (7) [37].

$$
\begin{aligned}
& x_{\text {intrm }}=\frac{x_{s}+x_{d}}{2} \\
& y_{\text {intrm }}=\frac{y_{s}+y_{d}}{2}
\end{aligned}
$$

Source node finds intermediate nodes which are above and below the reference axis midpoint node. Let $U_{23}\left(x_{\text {intrm }}^{\prime}, y_{\text {intrm }}^{\prime \prime}\right)$ and $U_{27}\left(x_{\text {intrm }}^{\prime \prime}, y_{\text {intrm }}^{\prime \prime}\right)$ be the location of intermediate nodes above and below the reference axis respectively. Where $x_{\text {intrm }}^{\prime}=x_{\text {intmm }}$ and $x_{\text {intrm }}^{\prime \prime}=x_{\text {intrm }}$ and $y_{\text {intrm }}^{\prime}, y_{\text {intrm }}^{\prime \prime}$ are computed using the equation (32) and (33) respectively.

$$
\begin{aligned}
& y_{i n t r m}^{s}=(n \times R)+y_{\text {intrma }} \\
& y_{\text {intm }}^{s}=y_{\text {intrm }}-(n \times R)
\end{aligned}
$$

Where $n$ and $R$ are degree of neighbour and communication range of sensor node respectively. Source node determines the ascending and descending angle for movement of mobile agent by using the equation (34) and (35).

$$
\begin{gathered}
\theta_{a 1}=\tan ^{-1}\left(\frac{y_{\text {intrm }}^{*}-y_{s}}{x_{\text {intrm }}^{t}-x_{s}}\right) \quad \text { if }\left(x_{\text {intmm }}^{*}\right)>x_{s} \\
\theta_{a 1}=2 \pi-\theta_{a 1}
\end{gathered}
$$

We consider $\theta_{d 11}=\theta_{d 1}$ and $\theta_{c 11}=\theta_{a 1}$ to be alternate angles as shown in figure (7).

Mobile agent (RDA) and its clone (replica of mobile agent) migrate for partial route discovery from source node to destination node on three routes as shown in figure (7). Mobile agents while migrating from one sensor node to next sensor node it collects the residual energy of node, distance between the nodes, hop count and deliver it to the destination node. Destination node uses this information to compute route weight factor and node disjoint routes.

\subsection{Establishment of Routes}

To find the best routes between the destination node and source node, the destination node computes node disjoint routes and route weight factor. It also determines the node disjoint routes by using the partial route discovered topology information. Two routes are said to be node disjoint, if and only if there is no common intermediate node between routes from source node to destination node. Destination node uses dijkstra algorithm to find the route based on the shortest distance. The nodes in the found route are deleted in the partial route discovered topology to find next route. The route 
finding continues until there is no disjoint route. A route weight factor is assigned to each route which is determined by using the equation(36).

$$
R^{w f}=\alpha_{1} R_{\text {eff }}+\alpha_{2} E_{\text {eff }}+\alpha_{2} H_{d f}
$$

Where $\Sigma_{i=1}^{3} \alpha_{1}=1$. Depending on the priority of the parameters the value of $\alpha_{i}$ can be chosen by the administrator. For example if energy efficiency is given more importance than $\alpha_{2}$ value will be higher.

\section{AGENCY}

The proposed work uses a set of static and mobile agents for route discovery and route computation, which are part of sensor node and destination node agencies. These are described as below.

\subsection{Sensor Node Agency}

The sensor node agency consists of Sensor Node Database (SNDB) for inter agent communication, Sensor Node Administration Agent (SNAA) and Route Discovery Agent (RDA). The interaction between the destination node agency components and its agents are depicted in figure (5).

Sensor Node Database: It resides in all the sensor nodes and it can be read and updated by the agents. It comprises of node id, distance between the neighboring nodes, residual energy, active/sleeping mode, destination node id, location information of destination node, upper and lower intermediate node location information, signal strength, available bandwidth, event sensing time, distance between itself and destination node, ascending and descending angles of intermediate nodes. The source node maintains the route/s information from itself to destination node as given by the destination node agency.

Sensor Node Administration Agent: It is a static agent resides in all sensor nodes of WSNs. It maintains the information available such as signal strength, residual energy, transmission range etc. It checks for event detection, if event is detected than it computes the arbitrary midpoint between the source node and destination node and intermediate nodes above and below the reference axis between source and destination node. It also computes the ascending and descending angle for agent movement. The SNAA also computes the route parameters like link efficiency, minimum mad maximum residual energy and hop distance factor. SNAA creates RDA and SNDB where it updates all the route information and node information.

Route Discovery Agent: It is a mobile agent resides in all the sensor nodes. Whenever a node detects the event than its SNAA generates RDA and its clone for route discovery. These RDAs migrates through the midpoint node which is on the reference axis, intermediate nodes above and below the reference axis with the help of ascending and descending angle to reach the destination node. These RDAs while migrating through the intermediate nodes they carry the information of relay nodes such as node id, residual energy, hop count, available bandwidth and hop distance; it also collects the neighbour information. After reaching destination node RDAs updates the DNDB.

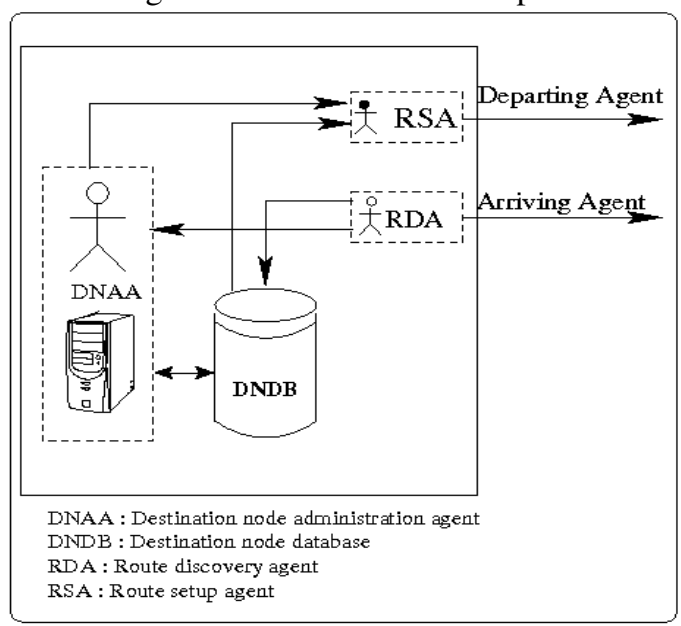

Figure 5: Sensor Node Agency 


\subsection{Destination Node Agency}

The destination node agency comprises of Destination Node Database (DNDB) for interagent communication, Destination Node Administration Agent (DNAA) and Route Setup Agent (RSA). The interaction between the destination agency components and its agents are depicted in figure (6).

Destination Node Database: It is the database which resides in the destination node and it can be read and updated by DNAA, RSA and RDA. It stores the network related information carried by RDA such as node id, residual energy, hop count, hop distance, minimum and maximum residual energy of each route, available bandwidth, channel capacity and DNAA information such as route weight factors, criticalness of event and number of available routes.

Destination Node Administration Agent: It is a static agent which resides in the destination nodes. It monitors the network related information such as number of active nodes, available route information to reach source node. It computes the route weight factors based on the collected information from RDAs such as route efficiency, route energy efficiency, hop distance factor and it also identify the node disjoint routes between destination node and source node than it priorities the routes based on their weight factor. Finally evaluates the criticalness of event, based on this it selects a single or multiple routes to source node and triggers the RSA to establish the routes to source node.

Route Setup Agent: It is mobile agent which resides in all the sink nodes. It is triggered by DNAA and it moves to source node by getting the required information from DNDB. After reaching the source node it updates the sensor node database by route information.

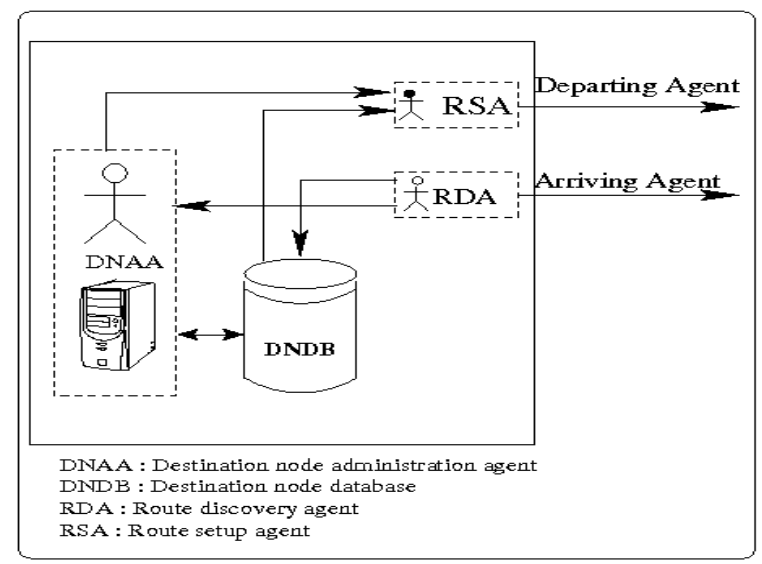

Figure 6: Destination Node Agency

\section{EXAMPLE SCENARIO}

This section describes the operation of proposed scheme. It comprises of anchor nodes (A1-A9), unknown nodes (U1-U81), source node (U40), destination node (D) and some agents for route establishment. The sequence operation of the scheme is as follows.

1. Whenever an event is detected at source node U40, it initiates the route discovery process to destination node.

2. SNAA computes the shortest route to destination node (U40-U28-U24-D), midpoint node U28, intermediate nodes above (U23) and below (U27) the reference axis and angles for agent movement.

3. SNAA generates RDA and its three clones. One RDA clone traverses on midpoint node and remaining two will traverses above and below intermediate nodes respectively by carrying the network, route and event related information.

4. RDA clones updates the DNDB after reaching the destination node (D).

5. DNAA computes the route weight factor and identifies the node disjoint routes and priorities them. 
6. DNAA evaluates the criticalness of event and selects multiple routes for reliable and robust communication as the event is noncritical.

7. DNAA triggers RSA; RSA generates three clones to establish the selected routes between destination node and source node.

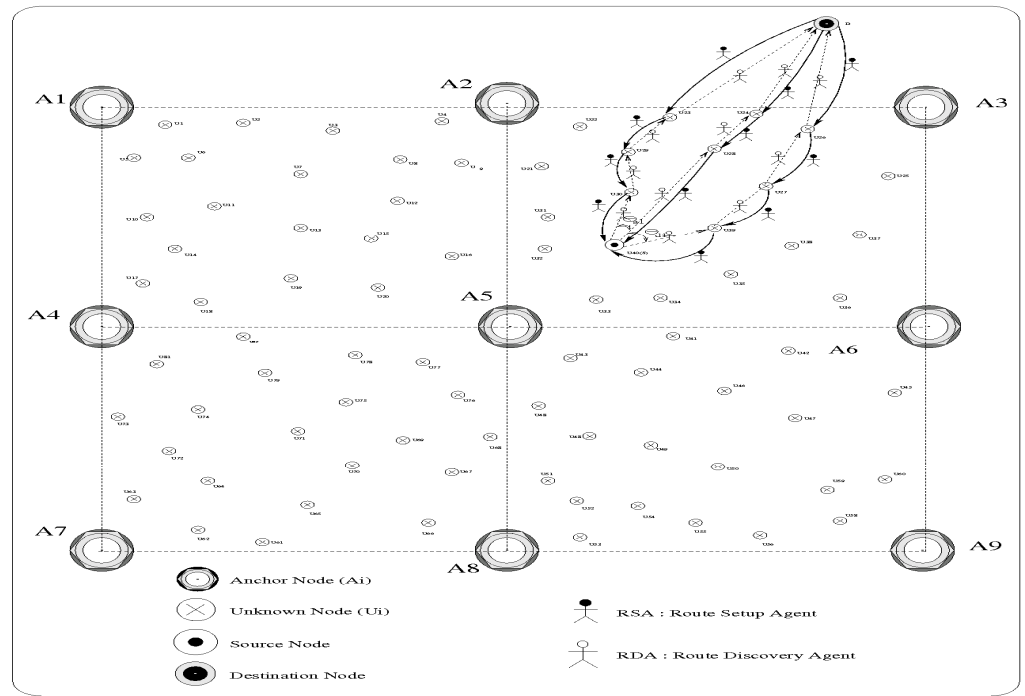

Figure 7: Example Scenario

\section{SIMULATION}

To test the performance of the proposed scheme, the scheme is simulated using $\mathrm{C}$ programming language. The proposed scheme is compared with an existing basic DV-hop localization scheme and agent based multipath routing scheme [33]. In this section we describe the simulation model, performance parameters and result analysis.

\subsection{Simulation Model}

Network model: We consider the network environment area of $l \times b$ square meters for WSN. A network consists of $N_{u}$ static unknown sensor nodes which are distributed randomly over the network environment and $N_{a}$ anchor nodes placed evenly over the network environment. The bandwidth of single hop connected sensor nodes is $B W_{\text {singlehep }}$.

Channel model: For the proposed scheme the S-MAC protocol is used for media access [38]. We assumed that packet transmission is in discrete time. Each node receives all packets from sending node during its receiving interval unless the sender node is in non-active state. We have assumed that channel is error free for simplicity.

Propagation model: For proposed scheme we used the free space propagation model with propagation constant $\beta$. The transmission range of each node in WSN is $\mathrm{R}$ for single hop distance. It is assumed that at any given time, the value of transmitted energy per packet is Eu Joules for every node. $\mathrm{R}$ is directly proportional to $\mathrm{Eu}$ of the node, that is, $\mathrm{R}=\mathrm{CEu}$, where constant of proportionality $\mathrm{C}$ depends on the medium of communication, attributed to $\beta$.

\subsection{Simulation Procedure}

Simulation parameters considered for analysing the scheme is given in table (1). Simulation procedure involves following steps.

1. Generate the network environment.

2. Apply the localization scheme.

3. Apply the proposed routing scheme.

4. Compute the performance parameters of localization and routing scheme. 
Table 1: Simulation Inputs

\begin{tabular}{|l|l|l|}
\hline Parameters & Notations & Value \\
\hline Length & $l$ & 5000 meters \\
\hline Breadth & $b$ & 5000 meters \\
\hline Number of nodes & $N$ & 200 \\
\hline Transmitting Nodes & $N_{t}$ & 40 to 200 \\
\hline Propagation constant & $\beta$ & 2.5 \\
\hline Sensor node communication range & $R$ & 300 to 500 meters \\
\hline Ascending angle threshold & $\theta_{\text {ath }}$ & $0^{\circ}-5^{\circ}$ \\
\hline Descending angle threshold & $\theta_{\text {dth }}$ & $0^{\circ}-5^{\circ}$ \\
\hline Initial energy of sensor node & $E_{f}$ & 1 KJoules \\
\hline Number of packets per seconds & $T r_{p k t s}$ & 256 per second \\
\hline Bandwidth per hop & $B W_{\text {single-hop }}$ & 5 Mbps \\
\hline Size of sensed data at each node & $S_{d}$ & 5 Kbytes \\
\hline Size of the processing code & $S_{\text {proc }}$ & 3 Kbytes \\
\hline
\end{tabular}

\subsection{Performance Parameters}

The following performance parameters are evaluated for analyses of the proposed schemes.

a. Localization error: It is defined as the ratio of summation of difference between the estimated and original position of unknown sensor nodes to the total number of nodes, which is given by the equation (37)

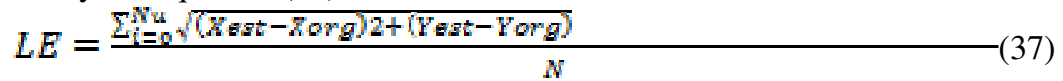

b. Network lifetime: It is the total number of rounds taken by the sensor node to die in the WSNs.

c. Cost factor: It is defined as the ratio of number of anchor nodes to the total number of nodes in the WSNs.

d. Energy consumption:

Energy consumption for localization: It is total amount of energy consumed to determine the location of all the unknown sensor nodes in WSNs.

Energy consumption for routing: It is total amount of energy consumed for data transmission, route discovery and route setup.

e. Packet Delivery Ratio (PDR): It is the ratio of packet sent to packet received.

f. Latency: It is the time required to transfer a packet from source node to destination sensor node.

g. Number of routes: It is the total number of node disjoint routes between the source node and destination node.

\section{RESULTS}

This section presents the results obtained during simulation. We compare the results of proposed scheme with existing basic DV-hop localization scheme and Agent Based Multipath Routing (ABMR) scheme for WSNs.

Localization Error: Figure (8) shows the localization error with increasing number of nodes and increasing number of anchor nodes. The proposed localization scheme provides the reduced localization error compared to basic DV-hop localization algorithm. As the number of nodes increases, each unknown node get a beacon packet with better received power which increases the localization accuracy their by reducing the localization error. As the number of anchor nodes increases the unknown node get increased number of beacon packets, so that the location accuracy of unknown nodes increases. 
Figure (9) describes the localization error versus number of nodes with uniform and random placement of anchor nodes. The proposed algorithm uses uniform placement of anchor nodes which reduces the localization error compared to random placement of anchor nodes. when the anchor nodes are placed randomly then some of the unknown nodes may not get the required number of beacon packets for localization which increases the localization error.

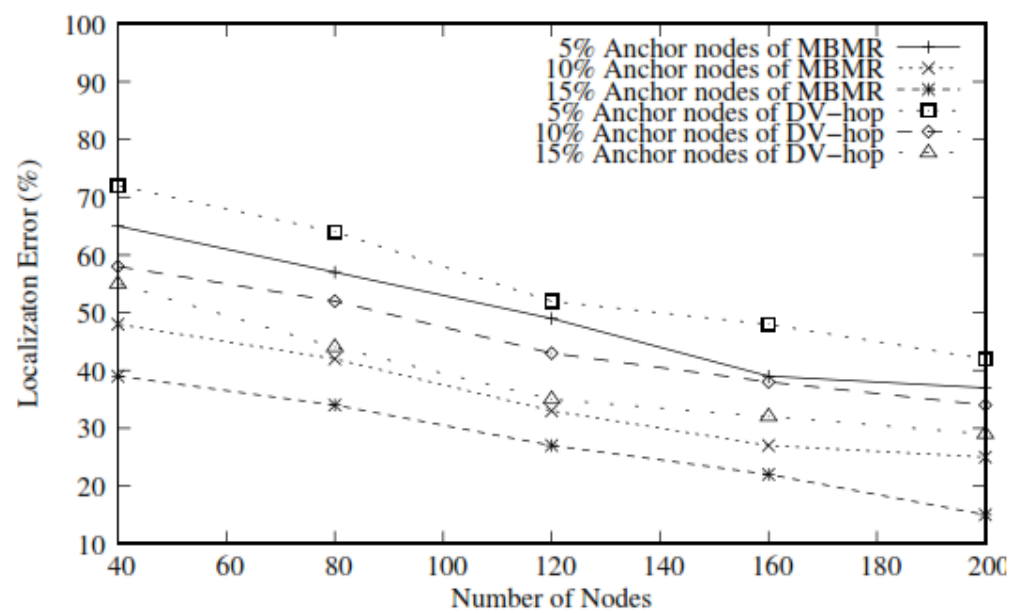

Figure 8: Localization Error Versus Number of Nodes

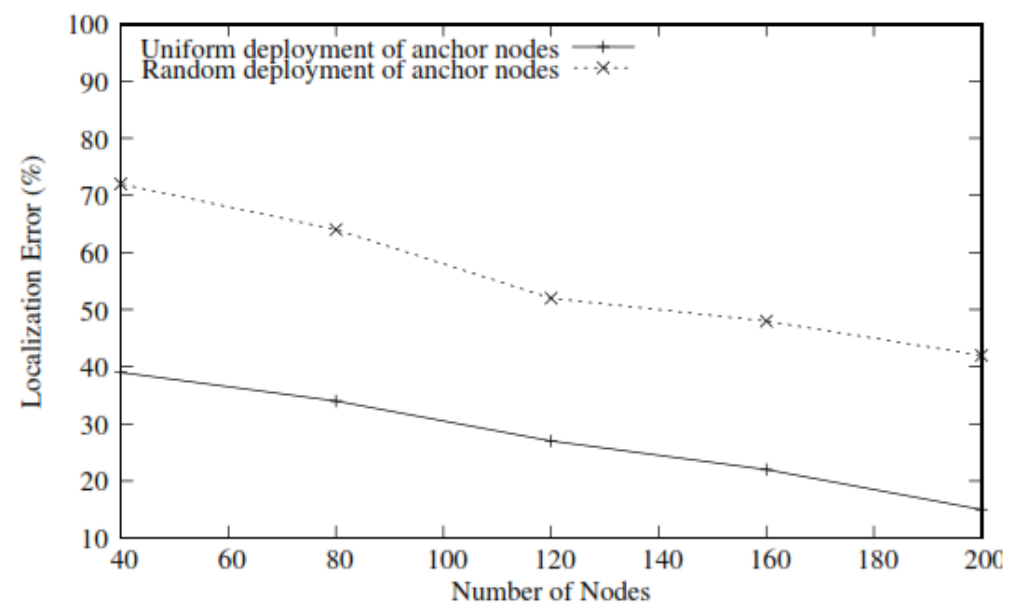

Figure 9: Localization Error Versus Number of Nodes with Uniform and Random Placement of Anchor Nodes

Network Lifetime: Figure (10) presents network lifetime for increasing number of nodes with and without GPS. As the number of nodes increases the hop distance for communication between the nodes reduces so that network lifetime of nodes increases as seen from simulation results. But in GPS equipped nodes GPS should continuously monitor the node location so it consumes a considerable amount of energy. Thus the proposed scheme uses very less number of nodes with GPS and remaining are without GPS to increase the network lifetime. 


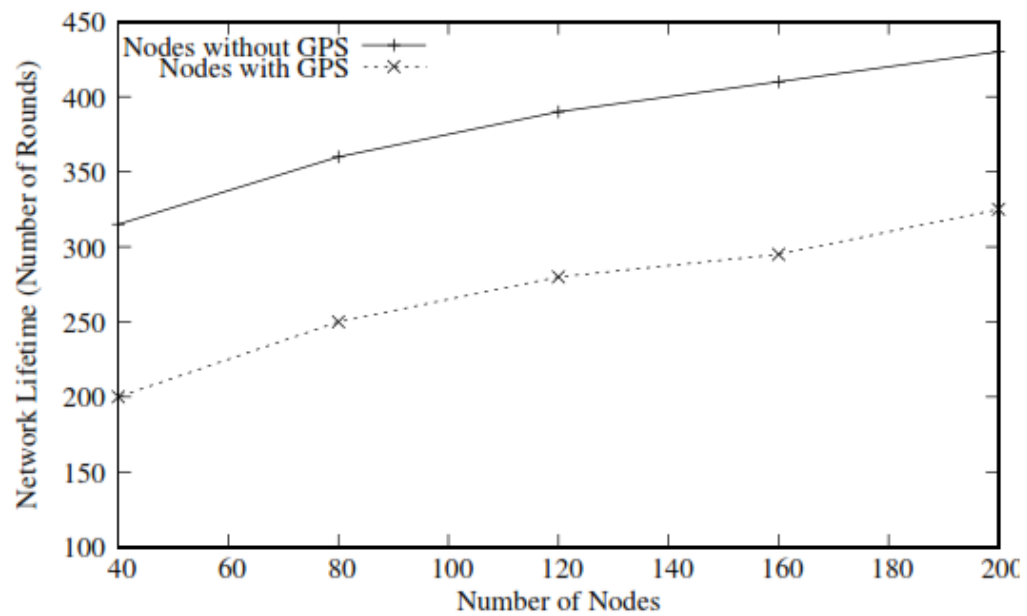

Figure 10: Network Lifetime Versus Number of Nodes

Cost Factor: Figure (11) shows cost factor with increasing percentage of anchor nodes. As the number of anchor nodes increases the cost of the network increases. Therefore to reduce the cost of the network the proposed scheme uses a very few number of anchor nodes. .

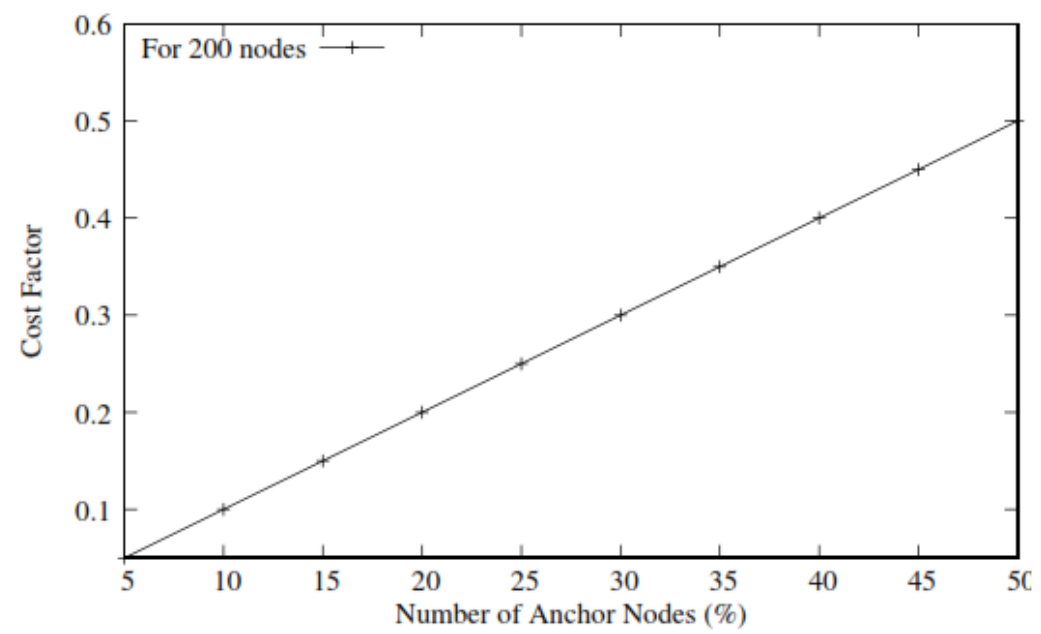

Figure 11: Cost Factor Number of Anchor Nodes (Percentage)

Energy Consumption: Figure (12) presents the energy consumption for localization in milliJoules with increasing number of nodes and increasing number of anchor nodes. As the number of nodes increases the energy consumption reduces because communication cost is reduced by smaller hop distances. But the computational cost increases which is negligible with respect to communication cost.

Figure (13) depicts the energy consumption for routing in milliJoules with increasing number of transmitting nodes and increasing communication range. As the number of nodes increases the energy consumption increases. However, the proposed MBMR performs better compared to ABMR. Energy consumption is due to gathering of partial topology information, route computation, route information transmission, and reception. The proposed MBMR scheme exhibits less energy consumption because it uses partial topology, hop distance factor, and energy information (energy efficiency) gathering for route computation. ABMR uses only topology information and hazard nodes for route computation. 


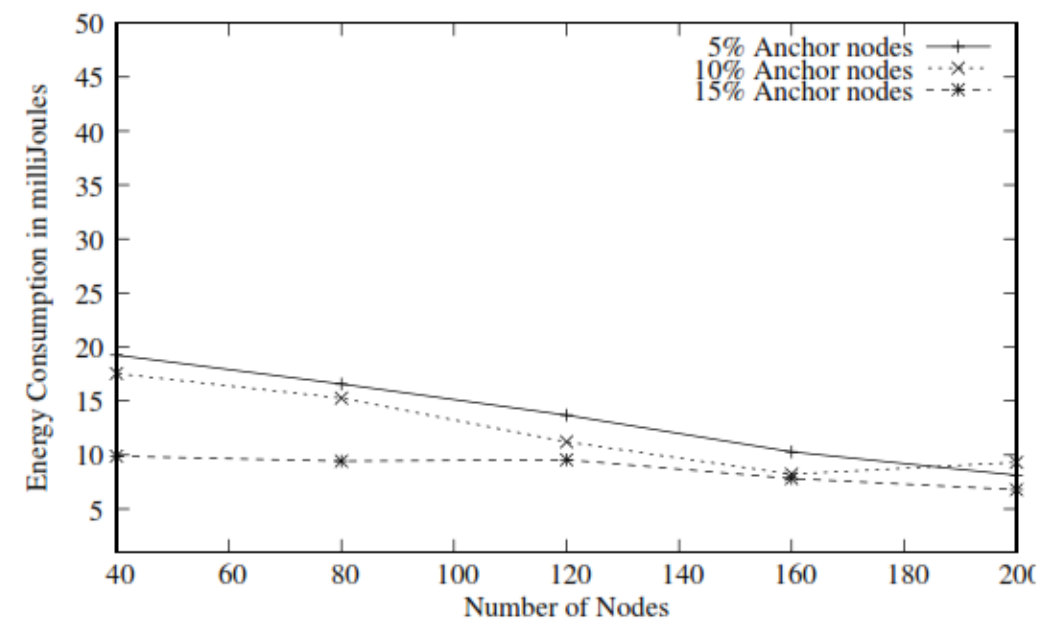

Figure 12: Energy Cosumption Versus Number of Nodes

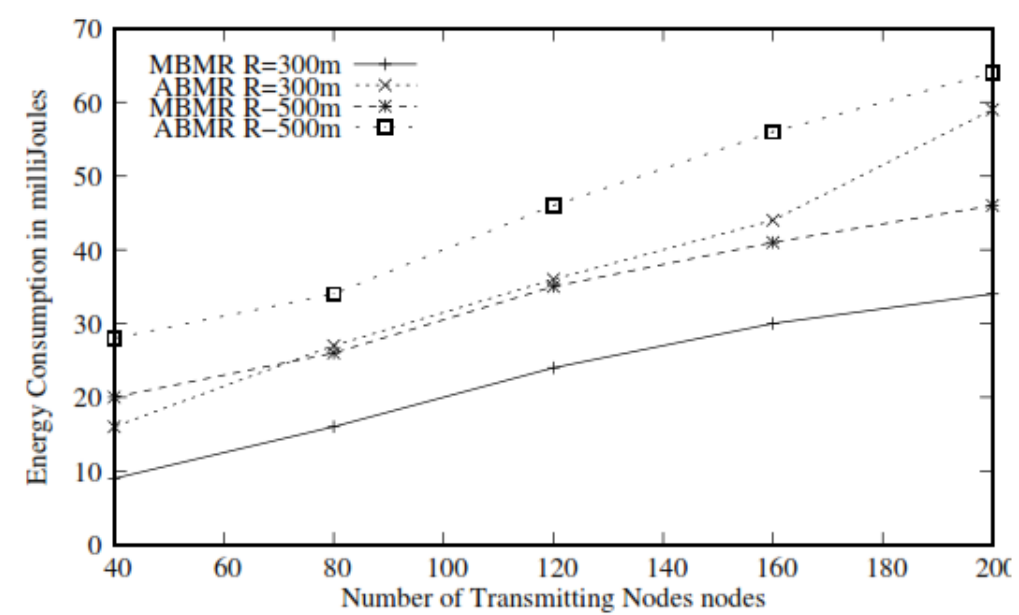

Figure 13: Energy Consumption Versus Number of Transmitting Nodes

Packet Delivery Ratio: Figure (14) depicts the packet delivery ratio with increasing number of transmitting nodes and communication range. As the number of nodes increases the PDR decreases and increase in communication range increases the PDR because it reduces the number of isolated nodes in the network. As the number of nodes increases, the data generated increases, which leads to lack of bandwidth therefore the PDR reduces. But the proposed MBMR scheme provides better PDR as compared to ABMR because it increases the bandwidth by considering link efficiency and establishing only required number of routes.

Latency: Figure (15) explains the latency for increasing number of transmitting nodes and increasing number of communication range. The proposed scheme performs better compared to ABMR. The latency is due to route information gathering, computation, transmission and reception. In ABMR the full topology information is used for route computation but in proposed MBMR the partial topology information is used to compute the node disjoint routes.

Number of Routes: Figure (16) depicts the number of node disjoint routes established for increasing number of nodes and increasing communication range. As the number of nodes increases, the number of node disjoint routes also increases and also increase in transmission range, increases the number of node disjoint routes. 
International Journal of Wireless \& Mobile Networks (IJWMN) Vol. 6, No. 2, April 2014

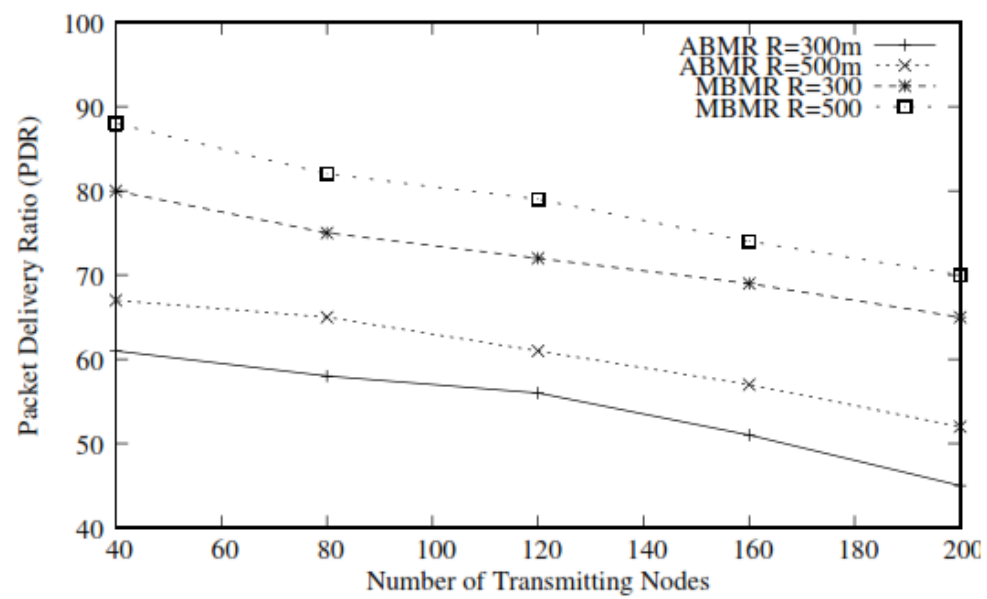

Figure 14: Packet Delivery Ratio Versus Number of Transmitting Nodes

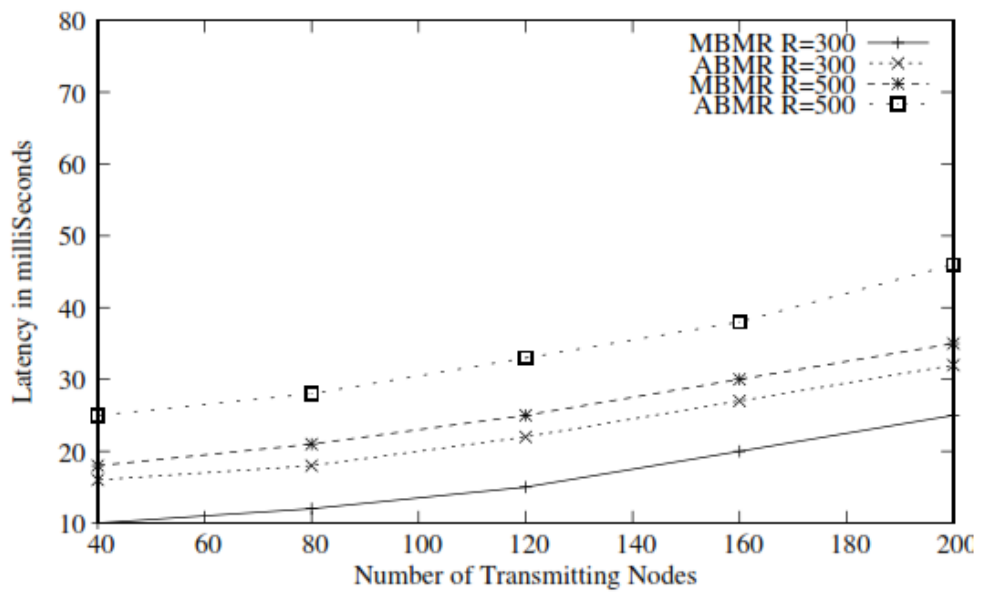

Figure 15:. Latency Versus Number of Transmitting Nodes

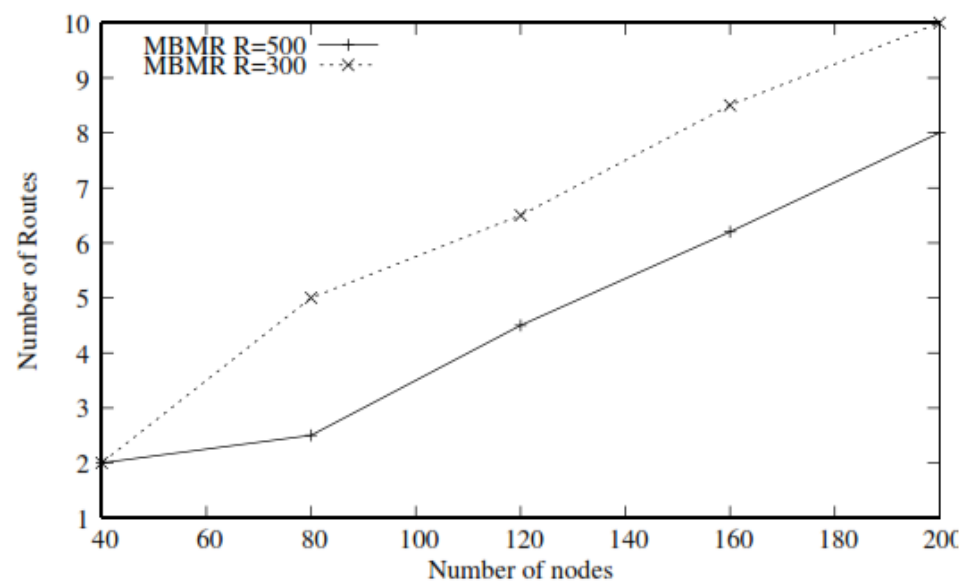

Figure 16: Number of Routes Versus Number of Nodes

\section{CONCLUSION AND FUTURE WORK}

We proposed the localization and multiagent based multipath routing scheme in WSNs.

In the first work, we proposed a localization scheme to reduce the energy consumption, size of sensor node and cost of network. The location of unknown node is determined by trilateration technique, by 
the reception of beacon packets from anchor nodes. The anchor nodes are placed uniformly throughout the WSNs to reduce the localization error.

In the second work, we proposed multiagent based multipath routing scheme in WSNs by employing localization technique, set of static and mobile agents. In the proposed scheme a source node triggers the route discovery mechanism by determining the intermediate nodes between source and destination node. The set of mobile agents are used to carry the partial topology information like node id, hop distance, residual energy and hop count etc., from source node to destination node through intermediate nodes by using location information. The partial topology information is used in computing the node disjoint routes by destination node. For node disjoint routes, the destination node computes the route weight factor based on the route efficiency, energy ratio and hop distance factor. For critical data, routing scheme assures intime data delivery by multiple routes; if non-critical then a single route with highest weight factor is established to assure reliable data transmission.

There is a good future for application of WSNs. Many of the WSNs applications make the layman job comfortable. But for these applications to become everyday reality a number of technological challenges need to be addressed. This section briefly explains the future work to proposed schemes.

For the proposed localization scheme, we can minimize the location error by employing any of the error minimization technique (soft computing technique). Also we consider the anchor nodes as mobile to reduce the location error. For the proposed routing scheme, we can consider some other geometry for better performance of routing scheme. We can also consider the multiple destination nodes or mobile destination node to improve the routing performance.

\section{REFERENCES}

1) Akyildiz, Ian F., et al., (2002) "A survey on sensor networks", Communications Magazine, IEEE 40.8, pp102-114.

2) Khemapech, I., I. Duncan, and A. Miller, (2005) "A survey of wireless sensor networks technology", 6th Annual Postgraduate Symposium on the Convergence of Telecommunications, Networking and Broadcasting.

3) Rahman, Kazi Chandrima, (2010) "A survey on Sensor Network", Journal of Computer and Information Technology, pp76-87.

4) Aboelaze, Mokhtar, and Fadi Aloul, (2005) "Current and future trends in sensor networks: a survey", Wireless and Optical Communications Networks, 2005. WOCN 2005. Second IFIP International Conference on. IEEE.

5) Akyildiz, Ian F., et al., (2002) "Wireless sensor networks: a survey" Computer networks 38.4, pp393-422.

6) Papageorgiou, Pavlos, (2003) "Literature survey on wireless sensor networks"

7) Pal, Amitangshu, (2010) "Localization algorithms in wireless sensor networks: Current approaches and future challenges", Network Protocols and Algorithms 2.1, pp45-73.

8) Langendoen, Koen, and Niels Reijers, (2003) "Distributed localization in wireless sensor networks: a quantitative comparison", Computer Networks 43.4, pp499-518.

9) Amundson, Isaac, and Xenofon Koutsoukos, (2009) "A survey on localization for mobile wireless sensor networks", Mobile Entity Localization and Tracking in GPS-less Environnments, pp235-254.

10) Pandey, Santosh, and Prathima Agrawal, (2006) "A survey on localization techniques for wireless networks", Journal of the Chinese Institute of Engineers 29.7, pp1125-1148.

11) Shang, Yi, et al., (2003) "Localization from mere connectivity", Proceedings of the 4th ACM international symposium on Mobile ad hoc networking \& computing.

12) Nasipuri, Asis, and Kai Li., (2002) "A directionality based location discovery scheme for wireless sensor networks", Proceedings of the 1st ACM international workshop on Wireless sensor networks and applications.

13) Niculescu, Dragos, and Badri Nath, (2003) "Ad hoc positioning system (APS) using AOA", INFOCOM 2003. Twenty-Second Annual Joint Conference of the IEEE Computer and Communications. IEEE Societies. Vol. 3.

14) Alippi, Cesare, and Giovanni Vanini, (2006) "A RSSI-based and calibrated centralized localization technique for Wireless Sensor Networks", Pervasive Computing and Communications Workshops, 2006. PerCom Workshops 2006. Fourth Annual IEEE International Conference on.

15) Bulusu, Nirupama, John Heidemann, and Deborah Estrin, (2000) "GPS-less low-cost outdoor localization for very small devices", Personal Communications, IEEE 7.5, pp28-34.

16) Priyantha, Nissanka B., et al., (2003) "Anchor-free distributed localization in sensor networks", Proceedings of the 1st international conference on Embedded networked sensor systems.

17) Rabaey, C. Savarese J., and Koen Langendoen, (2002) "Robust positioning algorithms for distributed ad-hoc wireless sensor networks", USENIX technical annual conference.

18) Huang, Tao, et al., (2010) "A Practical Localization Algorithm Based on Wireless Sensor Networks", Green Computing and Communications (GreenCom), 2010 IEEE/ACM Int'l Conference on \& Int'l Conference on Cyber, Physical and Social Computing (CPSCom).

19) Qian, Qingji, Xuanjing Shen, and Haipeng Chen, (2011) "An improved node localization algorithm based on DVHop for wireless sensor networks", Computer Science and Information Systems 8.4, pp953-972. 
International Journal of Wireless \& Mobile Networks (IJWMN) Vol. 6, No. 2, April 2014

20) Tian, Shuang, et al., (2007) "A RSSI-based DV-hop algorithm for wireless sensor networks", Wireless Communications, Networking and Mobile Computing. WiCom 2007. International Conference on. IEEE.

21) $\mathrm{Pu}$, Chuan-Chin, Chuan-Hsian $\mathrm{Pu}$, and Hoon-Jae Lee, (2011) "Indoor location tracking using received signal strength indicator", Emerging communications for wireless sensor networks, pp 229-256.

22) Kumar, Ram, N. Vinothraj, and G. Kiruthiga, (2012) "Energy Based Multipath Routing Protocols for Wireless Sensor Networks", Journal of computer application, Vol-5.

23) Rasouli Heikalabad, Saeed, et al., (2011) "QEMPAR: QoS and Energy Aware Multi-Path Routing Algorithm for Real-Time Applications in Wireless Sensor Networks", International journal of computer science issues, Vol-8.

24) Devisri, R., and R. J. Archana Devy, (2011) "Reliable and power relaxation multipath routing protocol for wireless sensor networks", Proceedings of international conference on advancement in information technology.

25) Ben-Othman, Jalel, and Bashir Yahya, (2010) "Energy efficient and QoS based routing protocol for wireless sensor networks", Journal of Parallel and Distributed Computing 70.8 pp849-857.

26) Malar, R. Then, (2010) "Congestion Control in Wireless Sensor Networks Based Multi-Path Routing In Priority Rate Adjustment Technique", proceedings of International Journal of Advanced Engineering \& Applications, pp28-33.

27) Li, Shuang, et al., (2010) "Efficient multi-path protocol for wireless sensor networks", International Journal of Wireless and Mobile Networks 2.1, pp110-130.

28) Pratheema, S., K. G. Srinivasagan, and J. Naskath, (2011) "Minimizing end-to-end delay using multipath routing in wireless sensor networks", International Journal of Computer Applications 21.5, pp20-26.

29) Chellaprabha, B., and S. Chenthur Pandian, (2012) "A Multipath Energy Efficient Congestion Control Scheme for Wireless Sensor Network", Journal of Computer Science 8, 2012.

30) Cherian, Mary, and T. R. Nair, (2012) "Multipath routing with novel packet scheduling approach in wireless sensor networks", arXiv preprint arXiv:1206.0420.

31) Liu, Wenjun, Shukui Zhang, and Jianxi Fan, (2012) "A Diagnosis-Based Clustering and Multipath Routing Protocol for Wireless Sensor Networks", International Journal of Distributed Sensor Networks.

32) Vidhyapriya, R., and P. T. Vanathi, (2007) "Energy efficient adaptive multipath routing for wireless sensor networks", IAENG International Journal of Computer Science 34.1, pp56-64.

33) Ramadan, Rabie A., (2009) "Agent based multipath routing in wireless sensor networks." Intelligent Agents, 2009. IA'09. IEEE Symposium on.

34) Sutagundar, A. V., and S. S. Manvi, (2012) "Wheel Based Event Triggered Data Aggregation and Routing in Wireless Sensor Networks: Agent Based Approach", Wireless Personal Communications, pp1-27.

35) Herbert Taub, Donald L. Schilling, (1986) "Principles of communication systems", McGraw-Hill.

36) Miao, Guowang, Nageen Himayat, and Geoffrey Ye Li., (2010) "Energy-efficient link adaptation in frequencyselective channels", Communications, IEEE Transactions on 58.2 pp545-554.

37) Sutagundar, A. V., and S. S. Manvi, (2013) "Location aware event driven multipath routing in Wireless Sensor Networks: Agent based approach", Egyptian Informatics Journal.

38) Heinzelman, Wendi Rabiner, Anantha Chandrakasan, and Hari Balakrishnan, (2000) "Energy-efficient communication protocol for wireless microsensor networks", System Sciences, 2000. Proceedings of the 33rd Annual Hawaii International Conference on. IEEE.

\section{Authors}

V. S. BENNUR completed master degree in digital communication, Visvesvaraya Technological University, Belgaum, Karnataka. Presently working as Assistant Professor for Department of Electronics and Communication Engineering, East Point Woman Engineering College, Bangalore. She is presently perusing her Ph.D in Visvesvaraya Technological University, Belgaum. Her area of interest includes network security, Wireless Sensor Networks, image processing, error control coding, etc., She published 5 national/international conference paper.

S. S. SHIRABUR obtained his bachelor degree in Electronics and Communication Engineering and Master degree in Digital Communication from Visveswaraya Technological University, Belgaum, Karnataka. He is currently working as an Assistant Professor for Department of Electronics and Communication Engineering, Veerappa Nisty Engineering College Shorapur, Karnataka, India. His area of interest includes Wireless networks, Multimedia Networks, Computer communication networks and Image Processing.

A. V. SUTAGUNDAR completed his Ph.D. from Visvesvaraya Technological University, Belgaum, Karnataka. Presently he is serving as an Associate Professor of Department of Electronics and Communication Engineering Bagalkot, Karnataka. His areas of interest include Signal and system, Digital Signal Processing, Digital Image Processing, Multimedia Networks, Computer Communication networks, Wireless networks, Mobile ad-hoc networks, Agent technology. He has published 26 papers in referred National/International Conferences and 11 papers in international journals.
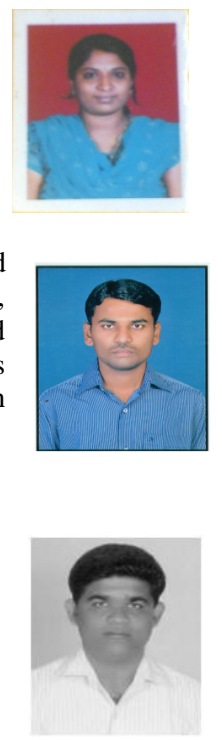